\title{
The Effect of Material Mechanics on Artificial Cartilage Defects Repaired in Tissue Engineering
}

\author{
Chun-Qiu ZHANG ${ }^{1}$, Xiang-Shuo WANG $^{2}$ and Tong-Tong GUO ${ }^{2 *}$ \\ ${ }^{1}$ College of Mechanical Engineering, Tianjin University of Technology Tianjin,300384,China \\ ${ }^{2}$ Harbin Institute of Technology Shenzhen, 518055, China \\ tongtong.guo@hitsz.edu.cn
}

Keywords: Tissue engineering, Bone material, Bone defect, Finite element.

\begin{abstract}
The stress distribution of artificial cartilage and host cartilage after repairing defect by tissue engineering was researched through finite element method under rolling compression loads. Taking knee articular cartilage as the research object, we established a three-dimensional finite element model of relative-rolling movement of articular cartilage. According to the dynamic boundary conditions between the femur and tibia during normal walking, finite element technique was used to analyze articular cartilages with different elastic module, different compression, different walking speeds and different defect sizes under the rolling compression load. The increases of both the elastic modulus of the implant and compression make the Mises stress increase in both artificial cartilage and host cartilage. The impact of different speeds and defect sizes were not obvious. The elastic modulus of the implant and compression have a more pronounced effect on Mises stress state of articular cartilage at the defect site after surgery. They are the main factors that worth being noticed in clinical treatment of cartilage defects and postoperative rehabilitation stage. Finite element dynamic analysis, which more closely simulates the force situation of repaired cartilage during walking, provides some theoretical reference for the repairing of articular cartilage defect by tissue engineering.
\end{abstract}

\section{Introduction}

Cartilage is difficult to self-repair after trauma or degeneration[1]. Treatment of cartilage defects all show different shortcomings in one way or another. Cartilage tissue engineering is becoming an ideal treatment to repair tissue defects permanently [2]. Tissue engineering construct cartilage tissue in vitro, and then is transplanted to the defect site to treat cartilage defects, which method has become the focus of orthopedic research[2].

After tissue engineered cartilage defects are implanted, the elastic modulus, implantation boundary and human motion of articular cartilage all affect the mechanical state of the defect, thus affecting the structure, shape of repair cartilage and metabolic state.

Abnormal mechanical state can cause artificial cartilage degradation, denaturation, junction cracking or sclerosis, which bring disaster to the host cartilage.

The study of the mechanical state after tissue engineering cartilage defects implanted is necessary. At present, a variety of mechanical measurement methods is difficult to achieve, so we are here using finite element method for research. Although some researchers have done a cartilage-related exploration, such as W. Wilson, B. van Rietbergen researched that how mechanical loading leads to cartilage damage and degeneration after meniscus is respected[3]; John $\mathrm{Z}$. Wu, Walter Herzog researched mechanical behavior of cartilage under the free compression[4]; Christopher Lovell Smith analyzed the process on repairing cartilage defect[5]. However, the study of the mechanical state of cartilage repair under the rolling load has not been reported. We used the finite element technique to establish the defect implantation repair model, and then analyzed the effect of elastic modulus, compression volume, rolling speed and defect type on the stress distribution of the repair site under the rolling load to defect site, so that to provide theoretical basis and reference information for clinical treatment of cartilage defects. 


\section{Material and Method}

\section{Finite Element Model}

According to the physiological state of articular cartilage, we used finite element software ABAQUS to establish a three-dimensional finite element model of the rigid cylinder that is rolling on a striped cartilage, and analyzed the mechanical situation of artificial cartilage and the surrounding host cartilage during the rolling process happening.

- Rigid cylinder: diameter $2 \mathrm{R}=30 \mathrm{~mm}$, length $8 \mathrm{~mm}$,

- Strip cartilage model: Articular cartilage thickness is usually $2 \sim 7 \mathrm{~mm}$. here we take the thickness of $4 \mathrm{~mm}$; the establishment of thickness, width and length of $4 \mathrm{~mm} \times 8 \mathrm{~mm} \times 60 \mathrm{~mm}$ of the strip cartilage model.

- Defect type: The cartilaginous cartilage with different cross-section of the groove to simulate the different size of the defect, including $2 \times 3$ ( $2 \times 3$ represents the defect is $2 \mathrm{~mm}$ deep and $3 \mathrm{~mm}$ wide), $3 \times 3,3 \times 6$ three cases.

\section{Material Properties}

Cartilage is a special kind of biological material. It has some function such as compression, buffer and so on[6]. Therefore, we can discuss the mechanical properties of the implanted materials and the influence of the joint mechanical environment. Host cartilage selected isotropic material properties, whose stress-strain nonlinear relationship is in Table 1; artificial cartilage elastic modulus values is in Table 2 .

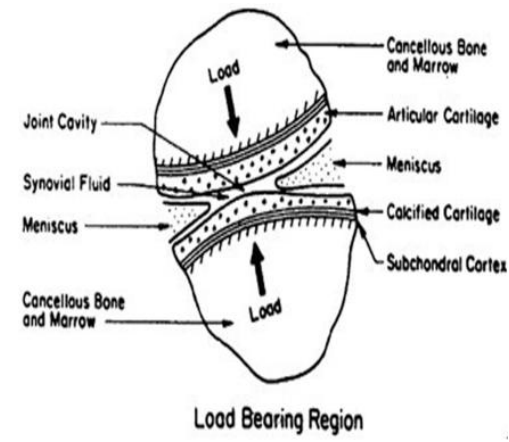

Fig. 1. Physiology structure of knee joint

Table 1.

\begin{tabular}{|c|c|c|c|c|c|c|c|c|c|c|}
\hline \multicolumn{11}{|c|}{ Non - linear stress - strain relationship of host cartilage } \\
\hline $\begin{array}{l}\text { stress } \\
(\mathrm{MPa})\end{array}$ & -0.15 & -0.08 & -0.02 & 0.00 & 0.13 & 0.30 & 0.48 & 0.67 & 0.87 & 1.10 \\
\hline Strain & 0.467 & 0.400 & $0 . \overline{233}$ & 0.000 & 3.330 & 6.670 & 10.000 & $\begin{array}{c}13.33 \\
0\end{array}$ & $\begin{array}{c}16.67 \\
0\end{array}$ & $\begin{array}{c}20.00 \\
0\end{array}$ \\
\hline $\begin{array}{l}\text { stress } \\
(\mathrm{MPa})\end{array}$ & 1.30 & 1.60 & 1.90 & 2.30 & 2.80 & 3.20 & 3.70 & 4.20 & 4.80 & \\
\hline strain & $\begin{array}{c}23.33 \\
0\end{array}$ & $\begin{array}{c}26.67 \\
0\end{array}$ & $\begin{array}{c}30.00 \\
0\end{array}$ & $\begin{array}{c}33.33 \\
0\end{array}$ & $\begin{array}{c}36.67 \\
0\end{array}$ & $\begin{array}{c}40.00 \\
0\end{array}$ & 43.330 & $\begin{array}{c}46.67 \\
0\end{array}$ & $\begin{array}{c}50.00 \\
0\end{array}$ & \\
\hline
\end{tabular}


Table 2.

Artificial cartilage elastic modulus / $\mathrm{MPa}$

\begin{tabular}{llll}
\hline 0.01 & 0.30 & 0.60 & 0.90 \\
\hline
\end{tabular}

\section{Contact and Boundary Conditions}

The upper surface of the cartilage and the outer surface of the cylinder is defined as the contact surface, whose friction coefficient is 0.02 ; the bottom of the cartilage is fully constrained and symmetrical on one side; with the rigid cylinder geometric center as reference point, we apply different rolling velocities:5 cm / s, $10 \mathrm{~cm} / \mathrm{s}$, and $15 \mathrm{~cm} / \mathrm{s}$ (angular velocity); in the Y direction we apply force $\mathrm{F}(\mathrm{N})$ that makes $5 \%, 10 \%, 15 \%, 20 \%$, and $30 \%$ of the thickness of normal cartilage.

\section{Result}

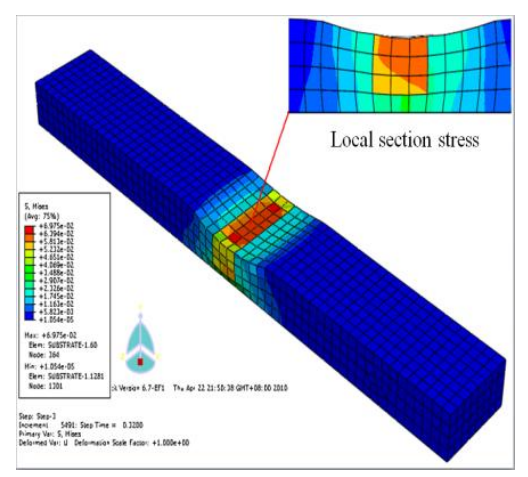

Fig. 2. The Mises stress distribution of the cartilage withrolling pressure above $3 \times 3$ defect

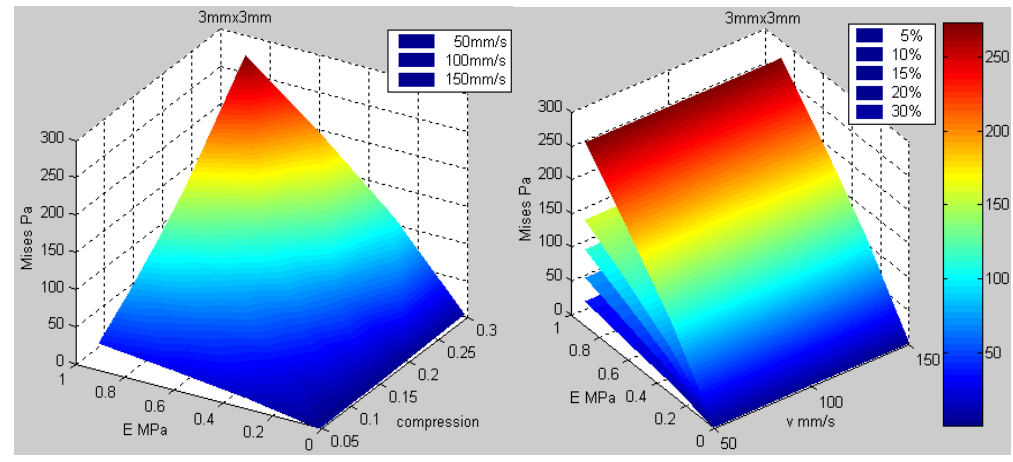

Fig. 3. The Mises stress of artificial cartilage with the modulus of elasticity and compression under different rolling speeds (left)

The change law of Mises stress of artificial cartilage with its elastic modulus and rolling velocity under different compression (right) 


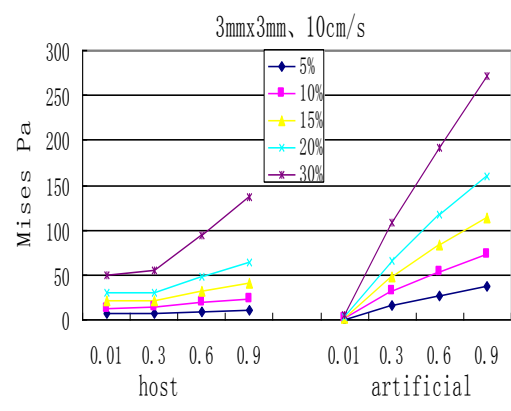

E MPa

a

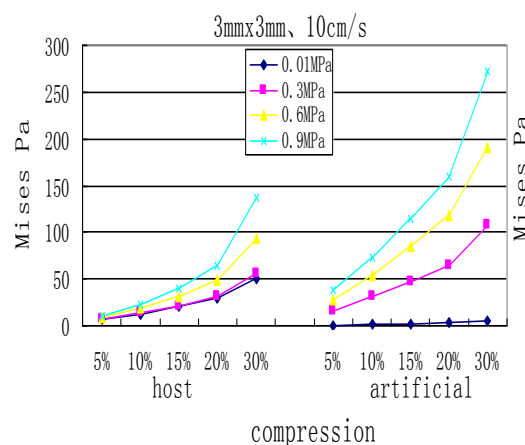

c

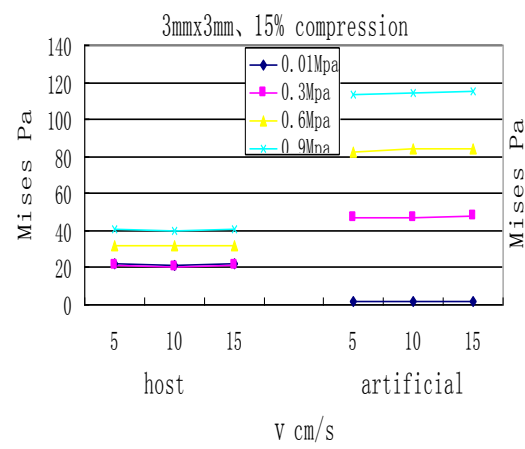

e

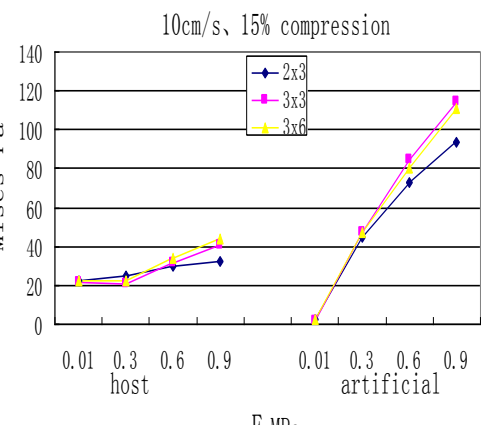

b

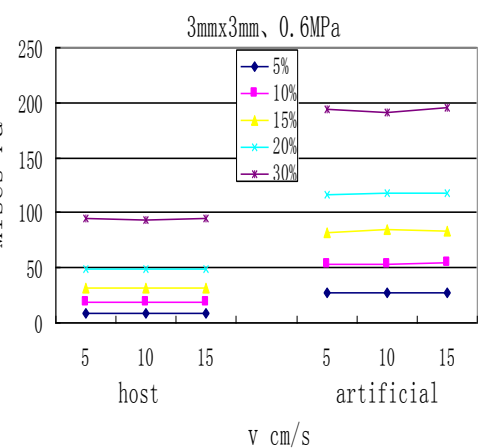

d

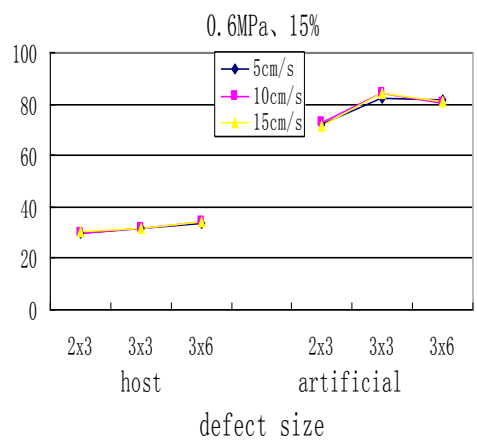

$\mathrm{f}$

Fig. 4. The Mises stress curve of elastic modulus of cartilage at different compression (a) and defect size (b)

The cartilage compression - Mises stress curve with different elastic modulus(c)

Cartilage velocity - Mises stress curve at different compression (d) and different elastic modulus(e)

The size of cartilage defect at different rolling speed - Mises stress curve(f)

Summarizing the influence of elastic modulus, compression volume, rolling speed and defect size on the Mises stress distribution of artificial cartilage and host cartilage during rolling load, the following four rules were obtained:

- The elastic modulus of artificial cartilage increased, the Mises stress of artificial cartilage and host cartilage increased at each compression volume and velocity and defect size(Fig. 3). The increasing trend of artificial cartilage was more obvious, and the force of artificial cartilage was larger than that of host Cartilage. When the elastic modulus of the artificial cartilage is high, the effect of size of the defect on the Mises stress begins to show (Figure 4, a, b). 
- With increasing of compression, the Mises stress of the defect of artificial cartilage and host cartilage increased at each speed, each elasticity modulus of artificial cartilage and each size of defects. The increasing tendency of artificial cartilage was more obvious, and the force of artificial cartilage was larger than that of host cartilage (Fig.4, c).

- When rolling speed increased, the Mises stress curves of artificial cartilage and host cartilage were basically consistent by each elastic modulus and defect size. in this simulation, the velocity range used had no obvious effect on them (Fig.4, d, e).

- When the depth and width of the defect were increased, the Mises stress of the artificial cartilage and host cartilage increased at each elastic modulus of artificial cartilage, each compression and each rolling speed.

\section{Discussion and Conclusion}

After repairing the tissue defects, if the defects in the host cartilage and artificial cartilage mechanical have different states, it will produce stress concentration and cracks in the joint surface, which would result in degradation of the original host cartilage and artificial cartilage. The simulation showed that the elastic modulus and compression of implanted cartilage have a significant effect on the Mises stress distribution at the postoperative defect, which is worthy of showing more attention in clinical treatment of cartilage defect and postoperative rehabilitation stage. However, the rolling velocity and the defect shape have no obvious effect on the Mises stress distribution of cartilage. Therefore, we should choose the appropriate material characteristics of artificial cartilage to implant and use reasonable exercise in the postoperative rehabilitation stage that could directly affect the stress distribution at the defect of cartilage, which is closely related to the success of the defect repair.

The results of the simulation show that the difference of Mises stress between artificial cartilage and host cartilage can reach a small value when the elastic modulus of artificial cartilage takes a certain value between $0 \mathrm{MP}$ and $0.3 \mathrm{MPa}$, and the two tend to be consistent, which is related to the material properties of host cartilage. According to the results, we could choose the best elastic modulus of artificial cartilage to implant for different cases. On the other hand, The interface in the simulation of artificial cartilage and host cartilage is idealized as completely bonding material, but the some cracks may exist in actually. This point needs to be studied in the future work.

\section{References}

[1] TIAN Tian. Application of mechanical loading in vitro with constructing tissue-engineered cartilage[J]. Chinese Journal of Aesthetic Medicine, Mar.2009. Vol.18.No3.

[2] Johnna S Temenoff, Antonios G Mikos. Review: tissue engineering for regeneration of articular cartilage[J]. Biomaterials 21 (2000) 431-440.

[3] W Wilson, B van Rietbergen. Pathways of load-induced cartilage damage causing cartilage degeneration in the knee after meniscectomy[J]. Journal of Biomechanics 36 (2003) 845-851.

[4] John Z Wu, Walter Herzog. Analysis of the mechanical behavior of chondrocytes in unconfined compression tests for cyclic loading[J]. Journal of Biomechanics 39 (2006) 603-616.

[5] Christopher Lovell Smith. Some aspects of the biomechanics of articular cartilage repair[D]. Department of Mechanical and Aerospace Engineering, CASE WESTERN RESERVE UNIVERSITY, the degree of Doctor of Philosophy, 2001.

[6] YAN Dan. Research advances in biochemical structure, biomechanical property and their relationship of articular cartilage[J]. Journal of Shanghai Jiaotong University(Medical Science), Vol.29 No.3 Mar. 2009. 
[7] Gerard A Ateshian, VikramRajan. Modeling the Matrix of Articular Cartilage Using a Continuous Fiber Angular Distribution Predicts Many Observed Phenomena[J]. Journal of Biomechanical Engineering, JUNE 2009, Vol.131.

[8] Christopher C-B Wang, Jian-Ming Deng, Gerard A Ateshian. An Automated Approach for Direct Measurement of Two-Dimensional Strain Distributions Within Articular Cartilage Under Unconfined Compression[J]. Journal of Biomechanical Engineering, OCTOBER 2002, Vol. 124. 